\title{
Idosos com câncer no período pré-operatório: dados de qualidade de vida, ansiedade e depressão
}

\section{Elderly with cancer in the preoperative period: data of quality of life, anxiety, and depression}

\author{
Anderson Lineu Siqueira dos Santos' (iD), Luceme Martins Silva² (iD), Zélia de Oliveira Saldanha² (iD) \\ ' Universidade do Estado do Pará, Centro de Ciências Biológicas e da Saúde, Programa de Pós-Graduação Stricto Sensu em Biologia Parasitária da \\ Amazônia, Belém, Pará, Brasil \\ ${ }^{2}$ Faculdade Cosmopolita, Curso de Enfermagem, Belém, Pará, Brasil
}

\section{RESUMO}

OBJETIVO: Avaliar dados da qualidade de vida (QV), a ansiedade e a depressão em idosos com câncer no período pré-operatório em um hospital de referência em oncologia de Belém, estado do Pará, Brasil. MATERIAIS E MÉTODOS: Trata-se de um estudo transversal, com 82 idosos com câncer que estavam internados em pré-operatório no Hospital Ophir Loyola, no período de abril a setembro de 2019. Os instrumentos utilizados na coleta de dados foram: questionário de qualidade de vida SF-36, Inventário Beck de Ansiedade e Inventário Beck de Depressão. RESULTADOS: Nos resultados para a avaliação da QV, considerando-se os scores correspondentes aos oito domínios do questionário SF-36, os aspectos sociais obtiveram a maior média. A totalidade dos participantes apresentou ansiedade moderada (79,3\%) ou severa $(20,7 \%)$. No entanto, a maioria (82,9\%) não apresentou qualquer grau de depressão. CONCLUSÃO: Concluiu-se que os scores de QV, ansiedade e depressão mostraram que é imprescindível uma atenção especial às subjetividades de idosos, considerando suas fragilidades de saúde.

Palavras-chave: Idosos; Câncer; Qualidade de Vida; Depressão; Ansiedade.

\begin{abstract}
OBJECTIVE: To evaluate quality of life (QOL), anxiety, and depression in elderly people with cancer in the preoperative period at a referral hospital in oncology in Belém, Pará State, Brazil. MATERIALS AND METHODS: This is a cross-sectional study with 82 elderly patients with cancer who were hospitalized in the preoperative period at the Hospital Ophir Loyola, from April to September 2019. The instruments used in the data collection were: SF-36 Health Survey questionnaire, Beck Anxiety Inventory, and Beck Depression Inventory. RESULTS: In the assessment of QOL, considering the scores corresponding to the eight domains of the SF-36 questionnaire, the social aspects obtained the highest average. All participants had moderate $(79.3 \%)$ or severe anxiety (20.7\%). However, the majority (82.9\%) did not present any degree of depression. CONCLUSION: Data of QOL, anxiety, and depression showed that it is essential to pay special attention to the subjectivities of the elderly, considering their health weaknesses.
\end{abstract}

Keywords: Elderly; Cancer; Quality of Life; Depression; Anxiety. 


\section{INTRODUÇÃO}

A Organização Mundial da Saúde (OMS) destaca que o câncer não precisa mais ser uma sentença de morte, pois existe a capacidade de reduzir a carga da doença e melhorar a sobrevivência e a qualidade de vida (QV) das pessoas portadoras de câncer? ${ }^{1}$.

câncer é uma doença crônico-degenerativa, caracterizada por um crescimento anormal e desordenado de células do corpo; de etiologia multifatorial e desencadeada por alterações genéticas, estilo de vida e fatores ambientais'. Para Camarano e $\mathrm{Kanso}^{2}$, a idade acima de 60 anos é considerada um fator de risco para o câncer; idosos possuem 11 vezes mais chances de desenvolver neoplasias que os adultos jovens. Foi estimada a ocorrência de cerca de 600.000 casos novos de câncer no Brasil em 2018 e, também, em $2019^{3}$

A QV é definida como um complexo conjunto de relações entre os domínios da vida, que englobam saúde física e psicológica, nível de independência, relações sociais, crenças pessoais e a relação do indivíduo com o meio ambiente ${ }^{4}$. Nos pacientes oncológicos, são observadas mudanças significativas na sua QV, devido a fatores que incluem desde mudanças físicas até emocionais, interferindo diretamente na conduta do paciente ao se submeter a procedimentos cirúrgicos ${ }^{5}$.

Lemos et al. ${ }^{6}$ demonstraram que o paciente no pré-operatório apresenta aumento de catecolaminas na corrente sanguínea, o que resulta em ansiedade, alterações da pressão arterial e na frequência cardíaca. Desse modo, a avaliação da ansiedade, baseada nas queixas do paciente, pode interferir na sua QV. Para Polanski et al. ${ }^{7}$ os sintomas de ansiedade e depressão estão relacionados à pior QV dos pacientes com câncer. A ansiedade está relacionada às limitações vivenciadas nessa fase do ciclo vital e que são, muitas vezes, interpretadas como ameaçadoras. A depressão apresenta impacto negativo na saúde dos idosos, por ter origens multicausais associadas a diferentes fatores que interagem e induzem de forma conjunta à patologia, diminuindo progressivamente a QV dos mesmos ${ }^{8}$.

No Brasil, estudos sobre a QV dos idosos em diferentes cenários são escassos $8,9,10$, portanto há necessidade urgente de atenção dos profissionais de saúde quanto à influência do envelhecimento na QV dos indivíduos.

Diante desse contexto, em pessoa idosa acometida por essa patologia, a atenção e o cuidado estão direcionados as suas necessidades e limitações. Desse modo, torna-se essencial adotar práticas assistenciais que estejam fundamentadas no bem-estar biopsicossocial e espiritual do indivíduo, a fim de proporcionar uma melhor $Q V^{1,4}$.

Assim, este estudo teve como objetivo avaliar os dados de QV, ansiedade e depressão em idosos com câncer no período pré-operatório em um hospital de referência em oncologia da cidade de Belém, estado do Pará, Brasil.

\section{MATERIAIS E MÉTODOS}

Trata-se de um estudo transversal quantitativo e descritivo, realizado com idosos em pré-operatório cirúrgico internados nas clínicas cirúrgicas do Hospital Ophir Loyola (HOL) em Belém. $\bigcirc \mathrm{HOL}$ possui 53 leitos de clínica oncológica, 48 leitos de cirurgia oncológica e serviços de assistência à oncologia pediátrica, quimioterapia, radioterapia e hematologia.

No presente estudo, foram incluídos indivíduos com idade igual ou superior a 60 anos, de ambos os sexos, com diagnóstico de câncer, internados em uma das clínicas cirúrgicas do referido hospital e que estivessem no período de pré-operatório. Foram excluídos idosos com diagnóstico não conclusivo para câncer, com déficit cognitivo ou apresentando algum distúrbio mental que dificultasse o fornecimento de informações. $\bigcirc$ total de participantes do estudo foi de 82 pessoas.

\section{COLETA DE DADOS}

A coleta dos dados ocorreu no período de abril a setembro de 2019. Foram avaliados os dados de QV, ansiedade e depressão e aplicado um questionário referente a dados sociodemográficos e clínicos, para traçar o perfil dos participantes. A QV foi avaliada pelo questionário SF-36 (Medical Outcomes Study 36-Item Short Form Health Survey) ${ }^{11}$, que dispõe de oito domínios: capacidade funcional, aspectos físicos, dor, estado geral de saúde, vitalidade, aspectos sociais, aspectos emocionais e saúde mental. $\bigcirc$ score para cada domínio é distribuído em um intervalo de 0 a 100. Para avaliar ansiedade e depressão, foram utilizados o Inventário Beck de Ansiedade (IBA) ${ }^{12}$ e o Inventário Beck de Depressão (IBD) ${ }^{13}$, respectivamente.

\section{ANÁLISES DE DADOS}

Os dados foram analisados no software Statistical Package for the Social Sciences (SPSS) v2.0. As variáveis sociodemográficas e clínicas foram analisadas por estatística descritiva - frequência absoluta e relativa, média, mediana e desvio padrão (DP) - e os instrumentos foram analisados de acordo com a regra estabelecida por Ciconelli et al. ${ }^{11}$, Quintão et al. ${ }^{12}$ e Gandini et al. ${ }^{13}$, para posterior análise estatística descritiva. Foi utilizado o coeficiente alfa de Cronbach, para estimar a confiabilidade dos questionários aplicados pela análise das respostas obtidas dos respondentes, apresentando uma correlação média entre as perguntas.

\section{ASPECTOS ÉTICOS}

presente estudo foi aprovado pelo Comitê de Ética em Pesquisa do Hospital Ophir Loyola, em 4 de abril de 2019, sob parecer $n^{\circ} 3.244 .884$, seguindo a Resolução 466/2012 do Conselho Nacional de Saúde. Obteve-se o Termo de Consentimento Livre e Esclarecido assinado por todos os participantes do estudo.

\section{RESULTADOS}

Dos 82 pacientes incluídos no estudo, 44 (53,7\%) pertenciam ao sexo masculino e, de acordo com a distribuição por idade, a maioria $(40 ; 48,8 \%)$ estava na 
faixa etária de 61 a 70 anos. A procedência da capital (município de Belém) foi a maior $(42 ; 51,2 \%)$; o ensino fundamental incompleto $(72 ; 87,8 \%)$ foi o grau de escolaridade predominante; $49(59,8 \%)$ eram casados; 61 (74,4\%) declararam-se católicos; 60 (73,2\%) eram pardos; e 49 (59,8\%) eram aposentados. A renda familiar de um salário mínimo foi declarada pela maioria dos entrevistados $(61 ; 74,4 \%)$. Quanto ao tipo de câncer, o de estômago foi o mais frequente $(14 ; 17,1 \%)$, seguido por pele $(11 ; 13,4 \%)$ (Tabela 1).

Os resultados da avaliação de QV constam na tabela 2, na qual os scores dos oito domínios do questionário SF-36 (capacidade funcional, aspectos físicos, dor, estado geral de saúde, vitalidade, aspecto sociais, aspectos emocionais e saúde mental) foram apresentados.

Os escores mais altos foram obtidos nos domínios: aspectos sociais, com a média de 82,62 e DP $=18,92$; saúde mental, com média de 75,61 e $D P=17,07)$; dor, com média de 69,60 e DP = 21,89; aspectos emocionais, com média de 69,51 e $\mathrm{DP}=42,30$. $\bigcirc$ alfa de Cronbach $(\alpha=0,88)$ mostrou a confiabilidade da escala de QV SF-36, e assim o questionário foi preciso e consistente.

Quando analisados os scores do IBA, foi verificado que somente $20,7 \%(17 / 82)$ apresentava ansiedade severa e a maioria, 79,3\% (65/82), ansiedade moderada. Em contraste, o IBD mostrou que a maioria, $82,9 \%$ (68/82), não era portadora de depressão (Tabela 3). O alfa de Cronbach mostrou que tanto o IBA $(\alpha=0,91)$ quanto o IBD $(\alpha=0,91)$ foram precisos e consistentes.

Aos participantes que foram diagnosticados com ansiedade e depressão, foi disponibilizado apoio psicossocial pela equipe de profissionais especializados do $\mathrm{HOL}$

\section{DISCUSSÃO}

Este estudo avaliou dados de idosos no período pré-operatório de câncer, internados no HOL; mas, por se tratar de uma amostra não probabilística, os resultados devem ser analisados com cautela.

De acordo com o Instituto Nacional de Câncer $(\text { INCA })^{3}$, em países desenvolvidos, a ocorrência do câncer tende a incidir de forma similar em ambos os sexos. Dos resultados obtidos neste estudo, houve predomínio no sexo masculino. $\bigcirc$ câncer de próstata é o segundo tipo de câncer que mais ocorre em homem, seguido por tumores de pele não melanoma, cuja incidência, em 2018, foi de 70,42/100.000 homens no Brasil e de 30,16/100.000 homens na Região Norte brasileira, tendo aproximadamente $70 \%$ das suas ocorrências em países desenvolvidos. É considerado um tipo de câncer com maior incidência em idosos, visto que $75 \%$ dos casos ocorre a partir dos 65 anos de idade $^{3}$. $\bigcirc$ câncer mais prevalente, neste estudo, foi o de estômago $(17,1 \%)$, seguido do câncer de pele $(13,1 \%)$ e de próstata (9,8\%). Segundo estimativas do INCA, no sexo feminino, os cânceres de pele não melanoma, mama e do colo uterino são os mais prevalentes ${ }^{3}$.
Tabela 1 -Características demográficas e socioeconômicas de idosos no período pré-operatório, atendidos no Hospital Ophir Loyola, de abril a setembro 2019, em Belém, Pará, Brasil

\begin{tabular}{|c|c|c|}
\hline \multirow{2}{*}{ Variáveis } & \multicolumn{2}{|c|}{ Total } \\
\hline & $N=82$ & $\%$ \\
\hline \multicolumn{3}{|l|}{ Sexo } \\
\hline Feminino & 38 & 46,3 \\
\hline Masculino & 44 & 53,7 \\
\hline \multicolumn{3}{|l|}{ Faixa etária (anos) } \\
\hline$=60$ & 7 & 8,5 \\
\hline $61-70$ & 40 & 48,8 \\
\hline $71-80$ & 29 & 35,4 \\
\hline$\geq 81$ & 6 & 7,3 \\
\hline \multicolumn{3}{|l|}{ Escolaridade } \\
\hline Ensino Fundamental incompleto & 72 & 87,8 \\
\hline Ensino Fundamental completo & 6 & 7,3 \\
\hline Ensino Superior Completo & 4 & 4,9 \\
\hline \multicolumn{3}{|l|}{ Estado civil } \\
\hline Solteiro & 11 & 13,4 \\
\hline Casado & 49 & 59,8 \\
\hline Convive junto & 2 & 2,4 \\
\hline Viúvo & 18 & 22,0 \\
\hline Separado & 2 & 2,4 \\
\hline \multicolumn{3}{|l|}{ Religião } \\
\hline Católica & 61 & 74,4 \\
\hline Evangélica & 16 & 19,5 \\
\hline Espírita & 2 & 2,4 \\
\hline Sem religião & 3 & 3,7 \\
\hline \multicolumn{3}{|l|}{ Procedência } \\
\hline Capital (município de Belém) & 42 & 51,2 \\
\hline Interior do Estado & 40 & 48,8 \\
\hline \multicolumn{3}{|l|}{ Raça } \\
\hline Branca & 15 & 18,3 \\
\hline Parda & 60 & 73,2 \\
\hline Negra & 7 & 8,5 \\
\hline \multicolumn{3}{|l|}{ Renda familiar (salário mínimo) } \\
\hline 0 & 2 & 2,4 \\
\hline$<1$ & 13 & 15,9 \\
\hline 1 & 61 & 74,4 \\
\hline 2 a 3 & 6 & 7,3 \\
\hline \multicolumn{3}{|l|}{ Ocupação } \\
\hline Agricultor & 6 & 7,3 \\
\hline Aposentado & 49 & 59,8 \\
\hline Autônomo & 4 & 4,9 \\
\hline Costureira & 2 & 2,4 \\
\hline Desempregado & 2 & 2,4 \\
\hline Do Lar & 10 & 12,2 \\
\hline Funcionário Público & 1 & 1,2 \\
\hline Pensionista & 2 & 2,4 \\
\hline Pescador & 4 & 4,9 \\
\hline Transporte & 1 & 1,2 \\
\hline Vigilante & 1 & 1,2 \\
\hline \multicolumn{3}{|l|}{ Tipos de câncer } \\
\hline Bexiga & 3 & 3,7 \\
\hline Cólon & 6 & 7,3 \\
\hline Esôfago & 3 & 3,7 \\
\hline Estômago & 14 & 17,1 \\
\hline Faringe & 4 & 4,9 \\
\hline Fígado & 2 & 2,4 \\
\hline Garganta & 1 & 1,2 \\
\hline Laringe & 1 & 1,2 \\
\hline Mama & 5 & 6,1 \\
\hline Olho & 4 & 4,9 \\
\hline Pele & 11 & 13,4 \\
\hline Pênis & 1 & 1,2 \\
\hline Pregas vocais & 1 & 1,2 \\
\hline Próstata & 8 & 9,8 \\
\hline Pulmão & 2 & 2,4 \\
\hline Reto & 4 & 4,9 \\
\hline $\operatorname{Rim}$ & 2 & 2,4 \\
\hline Sigmoide & 1 & 1,2 \\
\hline Tireoide & 6 & 7,3 \\
\hline Vesícula biliar & 3 & 3,7 \\
\hline
\end{tabular}


Tabela 2 - Dados sobre QV, por domínio, obtidos por meio do questionário SF-36, de idosos com câncer no período pré-operatório, internados no Hospital Ophir Loyola, de abril a setembro 2019, em Belém, Pará, Brasil

\begin{tabular}{|c|c|c|c|c|c|}
\hline Domínios & Mediana & Mínimo & Máximo & Média & $\pm \mathrm{DP}$ \\
\hline Capacidade funcional & 80 & 0 & 100 & 68,96 & $\pm 27,83$ \\
\hline Aspectos físicos & 25 & 0 & 100 & 41,46 & $\pm 44,31$ \\
\hline Dor & 64 & 0 & 100 & 69,60 & $\pm 21,89$ \\
\hline Estado geral de saúde & 47 & 10 & 72 & 44,63 & $\pm 14,70$ \\
\hline Vitalidade & 70 & 15 & 100 & 65,55 & $\pm 18,64$ \\
\hline Aspectos sociais & 88 & 25 & 100 & 82,62 & $\pm 18,92$ \\
\hline Aspectos emocionais & 100 & 0 & 100 & 69,51 & $\pm 42,30$ \\
\hline Saúde mental & 80 & 24 & 100 & 75,61 & $\pm 17,07$ \\
\hline
\end{tabular}

Tabela 3 - Distribuição dos scores do IBA e do IBD de idosos no período pré-operatório de câncer, internados no Hospital Ophir Loyola, de abril a setembro 2019, em Belém, Pará, Brasil

\begin{tabular}{lcc}
\hline \multicolumn{1}{c}{ Scores } & $\mathrm{N}=82$ & $\%$ \\
\hline Inventário Beck de Ansiedade & & - \\
Grau mínimo de ansiedade & - & - \\
Ansiedade leve & - & 79,3 \\
Ansiedade moderada & 65 & 20,7 \\
Ansiedade severa & 17 & \\
Inventário Beck de Depressão & & 82,9 \\
Nenhuma depressão & 68 & 9,8 \\
Depressão leve & 8 & 6,1 \\
Depressão moderada & 5 & 1,2 \\
Depressão grave & 1 &
\end{tabular}

Sinal convencional utilizado: - Dado numérico igual a zero, não resultante de arredondamento.

O fato da procedência dos idosos ser da capital Belém, onde a exposição a agentes de polvição, as alterações ambientais e o estilo de vida (tipo de moradia, acesso à dieta de qualidade, entre outros) podem ter implicações diretas no desenvolvimento das neoplasias apresentadas pelos mesmos ${ }^{14,15}$, impactando a qualidade de vida e a saúde desses indivíduos.

Outro fator importante que pode ter influenciado significativamente a QV dos idosos estudados foi a baixa escolaridade. Husson et al. ${ }^{16}$ identificaram que a baixa escolaridade está associada a comportamentos adversos a saúde e piores padrões do cuidado, o que leva o indivíduo a uma pior QV. O grau de conhecimento do indivíduo é um fator importante para a profilaxia e a conscientização na prevenção do câncer ${ }^{17}$.

Foi identificado que a maior parte dos participantes era casado ou possuía união estável, revelando que - apoio familiar ou a presença de companheiro(a) proporciona suporte emocional, psicológico e social ao portador de câncer; consequentemente, os que vivem sozinhos são mais suscetíveis a isolamento e depressão ${ }^{17,18}$. Dessa forma, os resultados de ansiedade moderada e de ausência de depressão encontrados neste estudo confirmam a importância da presença e participação dos familiares.

Dos pacientes analisados no presente estudo, a maioria declarou ter algum tipo de religião. Segundo alguns estudos ${ }^{4,19,20}$, a religiosidade desempenha um papel importante no enfrentamento dos estágios da doença, pois a crença religiosa contribui para a diminuição dos níveis de ansiedade e depressão durante o tratamento do paciente oncológico, melhorando sua QV. Considerando que 96,3\% dos pacientes declarou ter religião, a ansiedade moderada e a ausência de depressão encontradas podem ser resultado desse fator.

Os idosos apresentaram baixo poder aquisitivo, posto que a maioria declarou renda menor ou igual a um salário mínimo, fator que influencia diretamente a QV dos mesmos, por estar relacionado ao aspecto biopsicossocial. Mesmo o idoso aposentado que, teoricamente, possui autonomia financeira, não terá condições de cobrir os gastos com o tratamento oncológico ${ }^{4}$.

Segundo a OMS, ter $Q V$ na velhice corresponde ao maior nível de saúde nos aspectos físico, social, psíquico e espiritual'. A aplicação do instrumento SF-36, neste estudo, possibilitou avaliar a QV dos idosos internados no $\mathrm{HOL}$ em condições de pré-operatório, e os domínios com maior score de QV foram: aspectos sociais, saúde mental, dor, aspectos emocionais, capacidade funcional e vitalidade, mesmo que alguns desses pacientes tenham apresentado, em relação a esses domínios, sintomas, sentimentos, perfis comportamentais e prática de atividades físicas.

Os domínios que merecem atenção especial, por afetar a QV dos idosos avaliados, são estado geral de saúde e aspecto físico. O primeiro ocasionado, provavelmente, pela incidência de várias patologias e doenças crônico-degenerativas, além do próprio processo natural do envelhecimento; e o segundo, por impedir ou limitar práticas de atividade física e alimentação adequada, o que afeta o aspecto biológico/fisiológico, causando estresse e tensão no idoso ${ }^{21}$.

As mudanças físicas e psicossociais nesse período da vida do idoso interferem negativamente na sua QV e podem evoluir para o surgimento de alguns sintomas 
psíquicos, como irritabilidade, ansiedade, depressão e disfunção sexual ${ }^{8}$. No presente estudo, quanto a ansiedade e depressão, os resultados predominantes foram ansiedade moderada e ausência de depressão.

$\mathrm{Na}$ avaliação dos dados obtidos para os níveis de ansiedade, observou-se que todos os pacientes apresentaram níveis elevados, sendo a maioria, $79,3 \%$, classificada como ansiedade moderada e $20 \%$, como ansiedade severa. Lemos et al. ${ }^{6}$, ao investigar a associação do período pré-operatório de pacientes com câncer e níveis de ansiedade, descobriu que a ansiedade pré-operatória está relacionada às preocupações do paciente com a doença, a hospitalização e os tipos de cirurgias, uma vez que pessoas diagnosticadas com câncer expressam ansiedade pela luta da sobrevivência e um futuro incerto 22 .

\section{CONCLUSÃO}

Neste estudo, os idosos com câncer internados para intervenção cirúrgica apresentaram uma QV moderada, com destaque para os aspectos sociais. No entanto, nos aspectos físicos gerais de saúde, os pacientes apresentaram níveis baixos no quadro da QV, e, nessa situação, é imprescindível uma atenção especial às subjetividades do idoso, a fim de melhorar esse quadro.

Os níveis de ansiedade foram preocupantes, e a maioria dos idosos não apresentou qualquer grau de depressão, o que pode ser benéfico para o paciente que está com a saúde debilitada.

Assim, este estudo contribuiu com o conhecimento sobre aspectos sociais, físicos, emocionais e de saúde mental de idosos no período pré-operatório de cirurgia oncológica, por apresentar a necessidade de um planejamento em saúde mais especializado para melhoria da QV desses pacientes.

\section{CONFLITOS DE INTERESSES}

Os autores declaram não haver conflitos de interesse em relação à pesquisa a ser publicada.

\section{CONTRIBUIÇÃO DOS AUTORES}

Os autores participaram de todas as etapas de elaboração e revisão do manuscrito, desde a concepção do estudo até sua elaboração.

\section{REFERÊNCIAS}

1 World Health Organization. Cancer [Internet]. Geneva: World Health Organization; September 12, 2018 [cited 2019 jun 19]. Available from: https://www.who.int/news-room/fact-sheets/detail/ cancer.

2 Camarano AA, Kanso S. Envelhecimento da população brasileira: uma contribuição demográfica. In: Freitas EV, Py L, organizadores. Tratado de geriatria e gerontologia. 4. ed. Rio de Janeiro: Guanabara Koogan; 2016. p. 133-152.

3 Ministério da Saúde (BR). Instituto Nacional de Câncer José Alencar Gomes da Silva. Estimativa 2018: incidência de câncer no Brasil [Internet]. Rio de Janeiro: INCA; 2018 [citado 2019 set 20]. Disponível em: http://wwwl.inca.gov.br/ estimativa/2018/.

4 Toneti BF, Paula JM, Nicolussi AC, Sawada NO. Qualidade de vida relacionada à saúde de idosos com câncer em tratamento adjuvante. Rev Rene. 2014 nov-dez; 15(6): 1030-8.

5 Mansano-Schlosser TC, Ceolim MF. Fadiga em idosos em tratamento quimioterápico. Rev Bras Enferm. 2014 jul-ago;67(4):623-9.

6 Lemos MF, Lemos-Neto SV, Barrucand L, Verçosa N, Tibirica $E$. A informação no pré-operatório reduz a ansiedade pré-operatória em pacientes com câncer submetidos à cirurgia: utilidade do Inventário Beck de Ansiedade. Rev Bras Anestesiol. 2019 jan-fev;69(1): 1 -6.
7 Polanski J, Jankowska-Polanska B, Rosinczuk J, Chabowski M, Szymanska-Chabowska A. Quality of life of patients with lung cancer. Onco Targets Ther. 2016 Feb;2016(9):1023-8.

8 Ferreira AS, Bicalho BP, Neves LFG, Menezes MT, Silva TA, Faier TA, et al. Prevalência de ansiedade e depressão em pacientes oncológicos e identificação de variáveis predisponentes. Rev Bras Cancerol. 2016 out-dez;62(4):321-8.

9 Valadares MO, Vianna LG, Moraes CF. A temática do envelhecimento humano nos grupos de pesquisa do Brasil. Rev Kairos Gerontol. 2013;16(1);117-28.

10 Gutierrez BAO, Auricchio AM, Medina NVJ. Mensuração da qualidade de vida de idosos em centro de convivência. J Health Sci Inst. $2011 ; 29(3): 186-90$.

11 Ciconelli RM, Ferraz MB, Santos W, Meinão I, Quaresma MR. Tradução para a língua portuguesa e validação do questionário genérico de avaliação de qualidade de vida SF-36 (Brasil SF-36). Rev Bras Reumatol. 1999 mai-jun;39(3):143-50.

12 Quintão S, Delgado AR, Prieto G. Validity study of the Beck Anxiety Inventory (Portuguese version) by the Rasch Rating Scale model. Psicol Reflex Crit. 2013;26(2):305-10.

13 Gandini RC, Martins MCF, Ribeiro MP, Santos DTG. Inventário de Depressão de Beck - BDI: validação fatorial para mulheres com câncer. Psico-USF. 2007 jun; 12(1):23-31. 
14 Loomis D, Huang W, Chen G. The International Agency for Research on Cancer (IARC) evaluation of the carcinogenicity of outdoor air pollution: focus on China. Chin J Cancer 2014 Apr;33(4):189-96.

15 Aleksandrova $K$, Pischon $T$, Jenab $M$, Bueno-de-Mesquita HB, Fedirko V, Norat T, et al. Combined impact of healthy lifestyle factors on colorectal cancer: a large European cohort study. BMC Med. 2014;12:168.

16 Husson O, Mols F, Fransen MP, van de Poll-Franse LV, Ezendam NPM. Low subjective health literacy is associated with adverse health behaviors and worse health-related quality of life among colorectal cancer survivors: results from the profiles registry. Psychooncology. 2015 Apr;24(4):478-86.

17 Dugno MLG, Soldatelli JS, Daltoé T, Rosado JO, Spada P, Formolo F. Perfil do câncer de mama e relação entre fatores de risco e estadiamento clínico em hospital do Sul do Brasil. Rev Bras Oncol Clin. 2014 abr-jun;10(36):60-6.

18 Neris RR, Zago MMF, Ribeiro MA, Porto JP, Anjos $A C Y$. Experiência do cônjuge diante da mulher com câncer de mama e em quimioterapia: estudo de caso qualitativo. Esc Anna Nery. 2018;22(4):e20180025.
19 Mesquita AC, Chaves ECL, Avelino CCV, Nogueira DA, Panzini RG, Carvalho EC. A utilização do enfrentamento religioso/espiritual por pacientes com câncer em tratamento quimioterápico. Rev Latino-Am Enfermagem. 2013 mar-abr;21 (2).

20 King $M$, Llewellyn $H$, Leurent B, Owen $F$, Leavey $G$, Tookman A, et al. Spiritual beliefs near the end of life: a prospective cohort study of people with cancer receiving palliative care. Psychooncology. 2013 Nov;22(1 1):2505-12.

21 Lima FKSM, Pietsak EF. Saúde do idoso: atividade física, alimentação e qualidade de vida. Rev Extendere. 2016 jan-jun;4(1):49-62.

22 Reticena KO, Beuter M, Sales CA. Vivências de idosos com a dor oncológica: abordagem compreensiva existencial. Rev Esc Enferm USP. 2015 jun;49(3):419-25. 